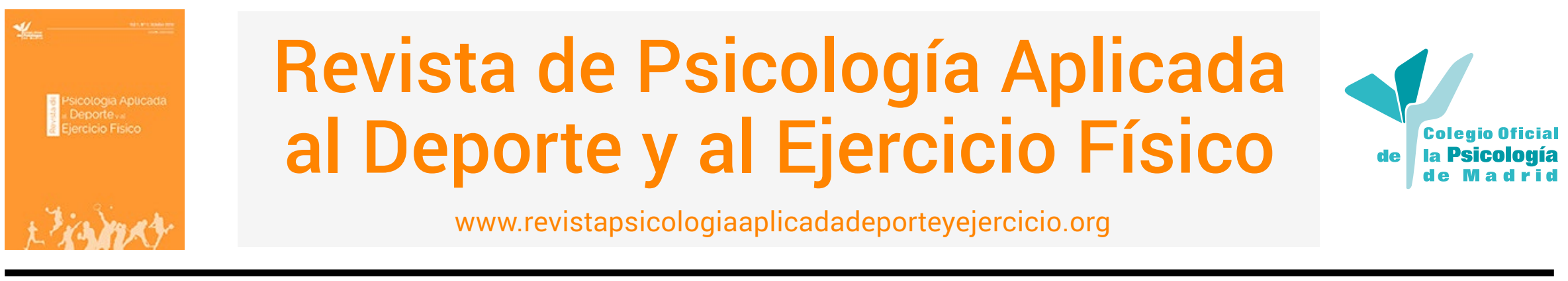

\title{
Un psicólogo en Catar: 13 años de experiencia profesional en Aspire Academy
}

\author{
Jaime Díaz-Ocejo \\ Qatar Football Association, Catar
}

RESUMEN: El objetivo del presente artículo es exponer los 13 años de experiencia que se ha desarrollado como psicólogo del deporte en Aspire Academy, en Catar. Se trata de una experiencia marcada por los distintos equipos de dirección por los que se ha pasado, provenientes de distintos países y enmarcada en una cultura tan diferente como la de Catar. Se describe la programación psicológica desarrollada en el atletismo a lo largo de los años, así como la metodología empleada en materia de captación de talentos deportivos desde la parcela de la Psicología del Deporte en Aspire. Los retos principales fueron lo concerniente a la barrera cultural, la carencia de instrumentos de medida baremados en lengua árabe, o la falta de experiencias y conocimiento previo en la literatura sobre el terreno aplicado en lo relacionado con el acompañamiento a jóvenes talentos hasta la élite. El resultado final fue la implementación de una programación de entrenamiento psicológico anual en el atletismo en Aspire. Se concluye que esta experiencia puede servir como ejemplo para profesionales que trabajen en el desarrollo de jóvenes deportistas en un entorno cultural diferente al occidental.

PALABRAS CLAVES: Deportistas árabes, desarrollo de talento, cultura.

\section{A psychologist in Qatar: 13 years of professional experience at the Aspire Academy}

ABSTRACT: The present article describes 13 years of experience working as a sport psychologist at the Aspire Academy, in Qatar. This experience has evolved around the different management teams, which came from different countries, and in a very different cultural environment such as that of Qatar. We describe the sport psychology program developed over the years in athletics, as well as our sport psychology approach to talent identification at Aspire. The main challenges were the cultural barriers, the lack of standardized measures in the Arabic language, and the absence of previous experiences and knowledge in the literature on applied practice in relation to talent development from grassroots to elite sport. The results evidenced the implementation of yearly psychological skills training in athletics at Aspire. In conclusion, this experience can serve as an example for practitioners working with young developing athletes in a cultural environment different from that of the West.

KEYWORDS: Arabic athletes, talent development, culture.

\section{Um psicólogo no Qatar: 13 anos de experiência profissional na Aspire Academy}

RESUMO: O objetivo deste artigo é expor os 13 anos de experiência enquanto psicólogo do desporto na Aspire Academy, no Qatar. É uma experiência marcada pelas diferentes equipas dirigentes com as quais trabalhou, provenientes de diferentes países e enquadrada numa cultura tão diferente como a do Qatar. A programação psicológica desenvolvida no atletismo ao longo dos anos é descrita, bem como a metodologia utilizada no recrutamento de talentos desportivos da área da psicologia do desporto na Aspire. Os principais desafios foram o referente à barreira cultural, a falta de instrumentos de medição medidos em árabe, ou a falta de experiência e conhecimento prévios sobre o campo aplicado em relação ao acompanhamento de jovens talentos até à elite. O resultado final foi a implementação

\footnotetext{
Jaime Díaz-Ocejo (iD https:// orcid.org/0000-0007-8254-5837 es psicólogo en la Selección Absoluta de Fútbol de Catar. Acreditado por la División de Psicología del Deporte y el Ejercicio, Sociedad Británica de Psicología, (British Psychological Society, BPS).

La correspondencia sobre este arículo debe enviarse a :Jaime Díaz-Ocejo. Aspire Academy/Football Performance Center (FPC), Aspire Zone street. P.O. box 22287. Doha, Qatar. E-mail:
} 
de um cronograma anual de treino psicológico no atletismo na Aspire. Conclui-se que esta experiência pode servir de exemplo para profissionais que atuam no desenvolvimento de jovens atletas num ambiente cultural diferente do ocidental.

PALAVRAS-CHAVE: Atletas árabes, desenvolvimento de talentos, cultura.

Artículo recibido: 03/03/2021 | Artículo aceptado: 16/05/2021

Todo comenzó con un anuncio de televisión durante el Campeonato del Mundo de Atletismo en Osaka, Japón, en el verano de 2007. En un breve instante de apenas dos segundos, apareció el logo de Aspire Academy en la pantalla, con un audio diciendo "Aspire Academy", sin más. Llamó tanto la atención al autor de este trabajo, que realizó una búsqueda en internet sobre Aspire Academy donde aparecía un departamento de Ciencias del Deporte con una unidad de Psicología del Deporte (PD en adelante). Tras entrar en contacto con la Academia por correo electrónico, se realizó una entrevista telefónica con uno de sus directores, Dieter Hackfort, quien por aquel entonces era Presidente del International Society of Sport Psychology, (ISSP). Al término de la entrevista, se le ofreció al autor una plaza de Senior Sport Psychologist y semanas más tarde aterrizaría en Doha.

Catar es un Estado soberano al este de la península arábiga y forma parte del Consejo de Cooperación para los Estados Árabes del Golfo, formado por el Reino de Arabia Saudita, Reino de Bahréin, Estado de Kuwait, Sultanato de Omán y Emiratos Árabes Unidos. Catar es una monarquía absoluta gobernada por la familia real Al Thani y gracias a sus exportaciones de gas natural líquido y petróleo goza de la renta per cápita mayor del mundo. En Catar, el 98\% de los musulmanes son Suníes y aunque la familia Al Thani está promoviendo la liberalización y modernización social, aún quedan cuestiones espinosas por resolver en materia de derechos humanos. Por ello, como se verá durante el desarrollo del artículo, la cultura representa el marco de referencia desde el que se ha desarrollado el desempeño profesional del autor y sobre la cual se abordarán algunos aspectos esenciales para una mejor compresión del mismo.

Trabajar en Aspire ha sido una experiencia única. Desde el principio es fácil verse desbordado por la cultura, el entorno físico y clima del país, las imponentes instalaciones deportivas, la multiculturalidad y las diferencias sociales. Esto era, más notable aún, en los primeros años de vida de Aspire. Catar es un país de contrastes, entre la modernidad y la tradición, donde pronto se aprende que el mejor aliado es el tiempo. Afortunadamente, las cosas van despacio en Catar, lo que permite ir poco a poco adaptándose al entorno social y laboral. Dado que el equipo de psicólogos del deporte siempre ha estado constituido por occidentales, así como la mayoría de los miembros del departamento de Ciencias del Deporte y entrenadores, la adaptación fue más llevadera, ya que ellos habían pasado por el mismo proceso de anteriormente.

Aspire Academy fue fundada por decreto del Emir de Catar en 2004 como parte de la estrategia deportiva de la "Visión Nacional de Catar 2030", a modo de institución independiente, aunque financiada por el gobierno de Catar. En 2008, Aspire se reestructuró en tres unidades estratégicas o strategic business units (SBUs); la propia Aspire Academy, Aspire Logistics (desde donde se organizan eventos deportivos como el Campeonato del Mundo de Atletismo en pista cubierta en Doha, en 2010, o conferencias de repercusión internacional como Doha Goals) y Aspetar, un hospital especializado en Medicina del Deporte acreditado por la FIFA. Los tres, bajo la denominación de Aspire Zone Foundation o AZF (Kilgallen, 2013). La mejor forma de presentar Aspire Academy sería como un centro de captación y desarrollo de talentos deportivos (edades de entre doce y dieciocho años) y que también cuenta con un centro de educación secundaria. Consta de dos pilares básicos en su programa; por un lado, el departamento de fútbol y; por otro, el de los denominados "deportes olímpicos" (atletismo, natación, tenis de mesa, squash, esgrima y golf), si bien es cierto que, aunque no todos los deportes que abarca este último departamento son olímpicos, en Aspire se les encuadra en el mismo. Los jóvenes deportistas pasan por tres fases de selección de pruebas físicas que determinarán quiénes entran finalmente en la Academia. El objetivo final es, pues, captar y desarrollar talento deportivo y formarles para una vez que se gradúen en el colegio, poder transferirles a las respectivas federaciones deportivas cataríes ya en categoría senior. Las instalaciones de la Academia son muy modernas y cuyo buque insignia es el Domo (Aspire Dome), el cual es considerado como la estructura cubierta más grande del mundo dedicada al deporte. Cuenta con un campo de fútbol, una piscina olímpica con plataforma de saltos, una pista de atletismo y varias canchas multideportivas, todo bajo un mismo techo. Igualmente, existen dos residencias para los deportistas. 
Afortunadamente, el número de experiencias aplicadas en PD continúa en progresión y está contribuyendo enormemente a nuestro enriquecimiento y formación como profesionales (Nigro y Androetto, 2020; Ruiz de Oña, 2018; Roffé 2016; García-Naveira, 2018; Morelló et al. 2018). Más específicamente en el atletismo, existen experiencias recientes sobre el desempeño profesional del psicólogo del deporte (García-Naveira, 2016), a través del coaching motivacional en la marcha atlética (Romero et. al, 2018), en la mejora del rendimiento deportivo (Jaenes y Caracuel, 2016; Díaz-Ocejo et al. 2013; Koehn y Díaz-Ocejo, 2016) o en cuestiones más relacionadas con la intervención en materia de salud mental (Jaenes et. al, 2019). En este artículo se presenta el trabajo personal de intervención realizado en PD con atletas de entre doce y dieciocho años en el atletismo en Aspire Academy entre 2008 y 2021. De un modo similar a lo apuntado por otros autores en el trabajo como psicólogo del deporte en el atletismo (García-Naveira, 2016), los objetivos han venido siendo fundamentalmente el fomentar el desarrollo del talento deportivo, promover resultados superiores y, lo que el autor considera más importante aún, cuidar del bienestar de los deportistas. A partir de estos objetivos generales y, como se verá en el procedimiento de la intervención, el objetivo principal ha sido el desarrollo e implementación de un programa de habilidades psicológicas para la mejora del rendimiento en el atletismo, el cual era, hasta el momento, inexistente en ese deporte en la Academia. No obstante, la intervención no ha estado exenta de dificultades relativas a la cultura -en cuanto a la complejidad de comprender, por ejemplo, las relaciones sociales-, la falta de instrumentos baremados en lengua árabe, o la carencia de diseminación científica relacionadas con la práctica de la PD aplicada con población infanto-juvenil. En ese sentido, son escasas las evidencias de psicólogos del deporte trabajando a tiempo completo y viajando a concentraciones y competiciones internacionales con deportistas adolescentes, especialmente en deportes como el atletismo y más concretamente en países con un nexo geográfico, cultural y religioso como los del del Consejo de Cooperación para los Estados Árabes del Golfo. A pesar del reconocimiento de esta carencia en otras culturas (Parham, 2005), la realidad es que existen muy escasas aportaciones a la literatura, siendo un ejemplo aislado la experiencia de Galloway (2009) con atletas de élite en Kuwait. Quizá, ello sea debido a que la psicología, en general, sigue siendo una tabú en la región, debido principalmente a que se sigue relacionando con "debilidades" que muchas personas aún no están dispuestas a compartir. Con eso en mente, el programa comenzó a constituirse con cautela y realmente priorizando la línea de lo que necesitaban los propios entrenadores.

\section{Etapas desde 2008 a 2021}

Para una mejor comprensión del desempeño profesional realizado, es menester contextualizar las distintas etapas a través de las cuales se ha ido desarrollando el mismo. En una primera instancia y desde el comienzo, entre 2008 y 2009, la dirección deportiva y de Ciencias del Deporte estaba a cargo de profesores de universidad y expertos en gestión deportiva alemanes. La PD formaba parte del departamento de Quality Management, Education and Social Affairs, (QESA), ubicado en el centro de educación de secundaria de Aspire y que contaba con cuatro psicólogos del deporte de nacionalidades diferentes. Por aquella época, el modelo de trabajo de Aspire entre deportistas, entrenadores, científicos del deporte y médicos del deporte era flexible y multidisciplinar. No existía una estructura predeterminada, así que todo iba a depender de la relación entre las distintas partes y la voluntad de cooperación mutua. La función del autor, básicamente, sería prestar servicios de PD aplicada en el atletismo, así como realizar evaluaciones psicológicas a todos los deportistas de la Academia dos veces al año, una labor realizada en colaboración con los otros psicólogos. Los compañeros estaban igualmente asignados a los distintos deportes respectivamente. En ese contexto, la experiencia previa del autor con atletas de la Federación Andaluza de Atletismo serviría como base para una mejor adaptación al entorno y desempeño en el equipo de atletismo de Aspire.

Entre 2010 y 2013, tras otorgársele a Catar la organización del Mundial de 2022, el departamento de fútbol se separó de los restantes deportes mayoritariamente individuales, los denominados "olímpicos", quedando la labor del autor final y únicamente centrado en el atletismo. En este período, la unidad de PD se reubicó bajo el departamento de Ciencias del Deporte, junto con Biomecánica, Fisiología del Ejercicio, Nutrición Deportiva y Preparación Física dentro de los aludidos deportes olímpicos. En esos años vinieron directores de Ciencias del Deporte y dirección deportiva de Australia, como por ejemplo Dennis Hatcher, antiguo director del Instituto Australiano del Deporte de Camberra (Australian Institute of Sport, AIS) quienes propusieron el "modelo australiano" al que hubo que adecuarse. Quizá, ya en este contexto puramente anglosajón, la adaptación del autor se vio atenuada por el hecho de ser Licenciado en Psicología en el Reino Unido y estar acreditado por la División de Psicología del Deporte y el Ejercicio de la Sociedad Británica de Psicología. Esto, al menos, le permitió "hablar el mismo idioma" en todos los sentidos con los nuevos compañeros. Lo más destacado del modelo australiano consistía en que giraba fundamentalmente entorno a equipos multidisciplinares de asesoramiento a deportistas y entrenadores -denominados service teams- donde 
se otorgaba gran relevancia a la figura del "Coordinador de Ciencias del Deporte", uno en cada sector del atletismo (saltos, velocidad y vallas, medio fondo y fondo y lanzamientos), tanta, casi, como al mismo entrenador. Se trataba de un sistema que había otorgado grandes resultados a Australia en los Juegos Olímpicos de Sídney (2000). Bajo este modelo, el concepto era que el entrenador "entrenaba", mientras que los científicos del deporte hacían de "navegador" de sus programas de entrenamiento. Con ello, se abandonaba el modelo al que Aspire se había adaptado hasta entonces, el modelo denominado en el mundo anglosajón como"coach-driven", donde la responsabilidad y dirección de cada equipo deportivo recaía en el entrenador. A partir de ahí, el Coordinador de cada service team haría de enlace entre el entrenador y los "científicos del deporte" o sport scientists y se encargaría de proponer reuniones cada dos semanas para compartir datos sobre los atletas entre todos. Bajo este modelo, se creó una estructura fija de científicos del deporte alrededor de cada entrenador; un fisiólogo, un biomecánico, un psicólogo, un nutricionista y un preparador físico.

Entre 2014 y 2017 entró una dirección de Ciencias del Deporte y gestión deportiva del Reino Unido, siendo el nuevo director de Ciencias del Deporte Tim Cable, también Presidente del European College of Sport Science (ECSC) por aquellos años. Se trataba de expertos en Ciencias del Deporte aplicado tras su reciente prestación en los Juegos Olímpicos de Londres 2012, como, por ejemplo, el coordinador de Ciencias del Deporte del equipo británico de atletismo o Team $G B$, Marco Cardinale. Lo primero que hicieron los jefes de las distintas unidades de Ciencias del Deporte, que ya eran en su mayoría británicos, así como el nuevo director técnico del atletismo, fue realizar un análisis de necesidades para entender la situación en Aspire. El objetivo era intentar mejorar ciertas metodologías en la Academia, como, por ejemplo, optimizar el conocimiento en la metodología empleada para la captación de talentos en Catar, mejorar la compresión del crecimiento y maduración de nuestros deportistas, o innovar en la optimización de la carga de trabajo para nuestra población infanto-juvenil. Con el objeto de comprender mejor todas estas áreas, se organizaron diversos congresos en Aspire para los que se trajeron a muchos de los mejores expertos internacionales. En 2014, se organizó un congreso sobre metodología de entrenamiento a estas edades (Youth Athletics Coaching Congress), otro en torno a los modelos de captación de talento deportivo (Aspire Talent Identification Conference) y en 2016, un último congreso dedicado a la carga de trabajo también en la población infanto-juvenil (Monitoring Training Loads, the Hows and the Whys).

En relación con la última etapa, desde 2018 hasta el primer semestre de 2021 y ya con la marcha de los directores británicos, hay que decir que ha supuesto una etapa más flexible en cuanto a la forma de trabajar y quizá sin una influencia proveniente de ninguna parte del mundo en concreto. Tal vez, una etapa ya marcada por un modelo "Aspire" propio. Aunque el autor ya había acompañado a algunos atletas junior de Aspire y a otros de categoría absoluta con la Federación de Atletismo de Catar (Qatar Athletics Federation, -QAF, en adelante-) a los Campeonatos del Mundo de Atletismo Junior en Bydgoszcz, Polonia en 2016 y Absoluto al aire libre en Londres 2017, a partir de aquí el desempeño profesional se centraría en la transición de la categoría junior a la absoluta. Una transición de junior a senior que es, posiblemente, la más complicada en la vida de un deportista. A partir de entonces ya no solo se prestarían servicios durante las concentraciones con los atletas de la Academia, sino también a atletas de élite de distintos sectores del atletismo de QAF. Para 2018, el objetivo era el Campeonato del Mundo de Atletismo Junior en Tampere, Finlandia y los Juegos Asiáticos en Yakarta-Palembang, en Indonesia. Para 2019, el Campeonato del Mundo de Atletismo de Doha y para el 2020 los Juegos Olímpicos de Tokio. Un aprendizaje fundamental durante esta transición en el trabajo con atletas junior hacia los de categoría absoluta durante esta etapa, tuvo que ver con la capacidad de adaptación rápida a distintos entrenadores, metodologías de trabajo, culturas -cada entrenador era de un país diferente- $y$, además, a contextos diferentes como eran los tan diversos países y centros de entrenamiento que se visitaban durante semanas. Por ejemplo, en febrero y marzo durante el tour europeo en pista cubierta de salto de altura en competiciones como las de Ostrava (República Checa) o Banska Bystrica (Eslovaquia) y los mítines de atletismo en verano como los de Merksem Antwerp Athetics Gala (Bélgica), o la Tuebingen Soundtrack Meeting (Alemania). Sin duda, convivir en el día a día con entrenadores y atletas en períodos largos de entrenamiento y competiciones requiere una habilidad adaptativa enorme a nivel de práctica profesional (Jaenes et. al, 2012). De hecho, en la línea de la filosofía de Mark De Rond (2012) y experiencia sobre su convivencia con el equipo de remo de Cambridge, lo cierto es que si se quiere ver como caza un tigre no se debe ir al zoo, se debe ir a su hábitat natural. Efectivamente, durante estos períodos, al principio supuso una adaptación importante a ese "hábitat natural", donde inicialmente se esperaba realizar un trabajo en un lugar en el que simplemente no era posible de la forma que se había anticipado. Es entonces cuando hay tener presente que los momentos clave del impacto que se puede tener como psicólogo del deporte pueden ocurrir en un aeropuerto, dando un paseo por cualquier ciudad donde se esté o en la recepción de un hotel, más que, en el caso del autor, en el propio Aspire. 


\section{Método}

\section{Participantes}

Los deportistas que forman parte de la Academia acceden con doce años y se gradúan con dieciocho. Dadas las particularidades culturales de Catar, la Academia está pensada solamente para chicos, algo que, aunque obviamente choca con la cultura occidental, en Catar está considerado como algo normal (algunas particularidades culturales serán abordadas en la sección dedicada a ello). El número habitual de alumnos evaluados cada año ronda los cinco mil, todos nacidos en Catar. Tienen once años cuando pasan por las pruebas de acceso para ver si son aptos para entrar en la Academia al año siguiente. Por lo tanto, estamos hablando de una población infanto-juvenil. En términos generales, suelen acceder alrededor de cincuenta chicos a la Academia para el curso siguiente en primero de educación secundaria.

\section{Materiales}

Esta sección merece una explicación para una mejor comprensión de los instrumentos elegidos en esta materia y que constan, por un lado, de dos pruebas psicotécnicas elegidas en formato computerizado y, por otro, de una entrevista breve. La forma de trabajar de la unidad de PD ha tenido desde sus comienzos como referencia el modelo Action Theory-Based Mental Test and Training System (MTTS en adelante), fundamentado en la Teoría de la Acción, de Nitsch y Hackfort (1981) y Hackfort et al., (2009). Este es un concepto que gira en torno a la plataforma computerizada de pruebas psicotécnicas y de biofeedback de Vienna Test System 2000Xpert, de la compañía Schufried (VTS en adelante).

El modelo MTTS propone que una vez que se miden ciertas capacidades cognitivas a través de la plataforma VTS, se puede manipular su entorno para entrenarlas en condiciones de laboratorio. De ese modo, se pueden introducir estímulos visuales de colores en pantalla y audio con sonido de público similar al de estadios de fútbol "crowd noise" o ruido monótono, "white noise", con el fin de entrenar la capacidad atencional, de toma de decisiones, de coordinación óculo manual o de procesamiento de información periférica en condiciones de distracción o demanda ambiental similares a las de la competición. El fin de este tipo de entrenamiento es que los aprendizajes se puedan extrapolar al contexto natural del deportista (Hackfort et. al.,2009). Algunas de las pruebas utilizadas en PD en Aspire son el Movement Detection Test (MDT), que mide la atención selectiva, velocidad de decisión y velocidad de respuesta motora, el Risk Choice (RISIKO), que mide la predisposición a asumir riesgos, el Two hand Coordination (2HAND), que mide la coordinación visuo-motora y manual, o el Peripheral Perception (PP), que mide la capacidad de procesamiento de la información periférica. Adicionalmente, los laboratorios de PD de Aspire también cuentan con tecnología de biofeedback de Vienna Test System 2000Xpert (respuesta galvánica de la piel, módulo de respiración diafragmática y electromiografía), lo cual proporciona la posibilidad de combinar la interacción entre la plataforma VTS con parámetros psicofisiológicos, opción que también contempla el modelo MTTS. La plataforma VTS viene siendo empleada en distintos trabajos en PD recientemente, como por ejemplo en deportes de equipo (De Andrade et. al, 2020; Kiss y Balogh, 2019; Schumacher et. al, 2018), de combate (Pulido-Pedrero et. al, 2020), con estudiantes de Ciencias del Deporte (Schumacher et. al, 2019), en deportistas de especialidades de tareas motoras abiertas y cerradas (Ong, 2017), o con población infanto-juvenil en nuestro contexto en Aspire (Díaz-Ocejo y Fountoulakis, 2011).

Dado que la realidad es que en el mundo árabe casi no existen pruebas de "lápiz y papel" baremadas en PD (aunque hay que decir que el inglés es lengua cooficial en Aspire), el concepto del modelo MTTS tiene su lógica en el contexto de la Academia. Además, aunque la mayoría de sus deportistas pueden comunicarse relativamente bien en inglés, tener las instrucciones en árabe en la plataforma VTS facilita la administración. Por otro lado, la plataforma VTS permite al deportista interactuar con ella, visualizando estímulos presentados en la pantalla, escuchando otros de tipo auditivo y respondiendo, pulsando los botones destinados para ello. Esto, por ejemplo, permite superar las limitaciones de evaluaciones con autoinformes al tratarse de pruebas objetivas computerizadas (Pulido-Pedrero et. al, 2020).

En primer lugar, las pruebas que se han venido utilizando en la fase de captación de talentos deportivos con la plataforma VTS son Cognitrone, que es una medida de concentración y Determination Test, que es una prueba de tolerancia reactiva al estrés en condiciones de estimulación compleja. Estas dos pruebas fueron elegidas como prioritarias dado el tiempo limitado con los candidatos y el consenso entre los psicólogos del deporte acerca de la relevancia de ambas capacidades en el contexto deportivo. Ambas fueron baremadas por la Unidad de PD de Aspire en 2009 para su población. En segundo lugar, se les realizaba una entrevista breve semiestructurada (de unos 10/15 minutos de duración) desarrollada por la Unidad de PD de Aspire, donde se exploran cuestiones relacionadas con la motivación, la percepción de competencia, los estresores y las estrategias de afrontamiento. Esta entrevista consta de las siguientes preguntas: 
a) ¿Por qué practicas deporte? La cual nos proporciona información sobre el origen y su motivación por la práctica deportiva o de un deporte en concreto.

b) ¿Cómo de bueno crees que eres en tu deporte? ¿En una escala del 1 al 10 -siendo el 1 la medida más baja y el 10 la más alta-, donde te situarías a ti mismo? Esta medida subjetiva nos da información sobre la percepción de competencia del joven deportista. De acuerdo con Horn (2004), en la preadolescencia parece existir una sobrevaloración de la percepción de competencia, que por otro lado parece ser deseable a estas edades. En caso contrario, examinamos con más en detenimiento el por qué durante la entrevista.

c) ¿Qué te estresa normalmente? Con esta pregunta indagamos las posibles fuentes de estrés y donde también aprovechamos para explorar sobre la situación familiar o de experiencias previas en competiciones deportivas.

d) ¿Qué haces cuando te sientes estresado? Aquí exploramos fundamentalmente si emplea alguna estrategia de afrontamiento o no (por ejemplo, de aproximación o evitación; de corte cognitivo o conductual; o simplemente no se afronta).

En realidad, esta información ha venido sirviendo como un screening inicial del perfil de rendimiento del deportista. Además, más importante aún para el desempeño profesional, ha servido como una primera toma de contacto y punto de partida para el trabajo con aquellos que finalmente son aceptados en Aspire.

\section{Procedimiento}

Se aborda este apartado desde dos puntos relevantes para la práctica profesional como psicólogos del deporte en Aspire. Por un lado, dado que uno de los objetivos de Aspire Academy es la captación de talentos deportivos, se explicará de qué manera ha contribuido la Unidad de PD a esa parcela. Por otro lado, se presentará la programación de la preparación psicológica específicamente desarrollada para el atletismo, al ser sobre la que ha girado mayormente el desempeño profesional del autor.

Las fases de selección y captación de talentos deportivos son llevadas a cabo por el departamento de Identificación de Talentos Deportivos o Talent Identification Department (TID). Las fases son tres; una primera entre octubre y noviembre, denominada Bronze Phase, una segunda en el mes de enero denominada Silver Phase y una última en marzo, denominada Gold Phase. La primera fase o Bronze Phase tiene lugar en los colegios públicos de Catar, mientras que las dos últimas se realizan en Aspire. En la primera fase se realizan pruebas físicas en los colegios que sirven de screening inicial, para lo que se desplazan entre cinco y diez técnicos de TID y entrenadores para llevar a cabo las pruebas, con material propio de Aspire, con el objeto de mantener la fiabilidad de las medidas. Se trata de pruebas físicas basadas en la Educación Física (por ejemplo; salto a pies juntos, lanzamiento de balón medicinal y medidas antropométricas de estatura, peso y extensión de brazos). Las pruebas físicas realizadas durante la segunda fase o Silver Phase en enero se llevan a cabo en Aspire y son más específicas a los deportes "olímpicos" de la Academia (por ejemplo; carrera de velocidad, salto de longitud, lanzamiento de pelota o tareas de precisión en esgrima). En la fase final o Gold Phase, los entrenadores de los distintos deportes "ojean" a los candidatos que quedan tras superar las dos fases anteriores y ya lo hacen a través de la observación durante la práctica de pruebas de atletismo, natación, tenis de mesa, squash, esgrima y golf. Finalmente, serán elegidos los candidatos sobre los que haya consenso entre los técnicos del TID y los entrenadores.

En cuanto a la contribución desde la PD en materia de captación de talentos deportivos, hay que decir que tan solo se participa en la última y definitiva fase de esta en marzo (tabla 1). No obstante, al contrario de lo que ocurre con las pruebas físicas que sirven como cribado en la detección de talentos, siempre se consensuó entre los psicólogos del deporte que desde una perspectiva ética la PD debería servir como herramienta de "inclusión" en el deporte infanto-juvenil y no de "exclusión" (Abbott y Collins, 2002). Es decir, la información proporcionada en relación con el perfil de rendimiento psicológico de cada candidato al departamento de Identificación de Talentos Deportivos de Aspire quedaría en tan sólo eso, información suplementaria para una mejor compresión del candidato. Esto es, en ningún caso con la finalidad de excluirle. De ese modo, la planificación de la última fase de captación de talentos incluye el screening del perfil psicológico a través de los instrumentos descritos en la sección de instrumentos (dos pruebas en la plataforma computerizada VTS y una entrevista). Los candidatos son acompañados desde el Aspire Dome -que es donde se llevan a cabo las últimas pruebas físico-deportivas de selección-, por un técnico del departamento de TID a la Unidad de PD. Allí, son recibidos por los psicólogos, quienes comienzan con la entrevista y más tarde la evaluación psicotécnica en la plataforma VTS. Existen cuatro estaciones dispuestas para ello, con el fin de agilizar el screening, durando el mismo una media de veinte a veinticinco minutos por candidato. Habitualmente esta última fase tiene lugar en dos días consecutivos.

En cuanto al procedimiento de la programación psicológica anual, siempre se tuvieron en consideración las necesidades de los entrenadores y lo que ellos pensaban que era 
Tabla 1. Fases del proceso de Captación de Talentos Deportivos en Aspire Academy y ubicación del screening llevado a cabo por la Unidad de Psicología del Deporte en el mismo

Cronograma de fases de pruebas de Captación de Talentos Deportivos en Aspire Academy

\begin{tabular}{cccc}
\hline Fase & Bronze Phase (octubre/noviembre) & Silver Phase (enero) & Gold Phase (marzo) \\
\hline Tipo de pruebas & Pruebas basadas en la Educación & Pruebas específicas a los deportes de & $\begin{array}{c}\text { Observación durante la práctica de de- } \\
\text { portes de Aspire y screening del perfil de } \\
\text { rendimiento psicológico }\end{array}$ \\
\hline Lugar & Colegios públicos & Aspire Academy & Aspire Academy \\
\hline
\end{tabular}

relevante desde la contribución en materia psicológica con sus atletas. Ello llevó a ir configurando una programación semanal y mensual acorde con el contexto de entrenamiento o competición en que el estarían inmersos en cada momento. Al final, tras años de implementación y desarrollo, se fue fraguando una programación anual aplicada y consensuada con los entrenadores. De igual modo, al tratarse de adolescentes de entre 12 y 18 años, sin experiencia previa en $\mathrm{PD}$, se estimó que el mejor abordaje sería desde una faceta educativa y pedagógica adecuada. Afortunadamente, se fue contando poco a poco con más apoyo por parte de los distintos entrenadores, a pesar de algunas reticencias iniciales por parte de alguno de ellos. Algo, por otro lado, como sabemos habitual en la PD aplicada. Para el tercer año, tras mucha paciencia, ya se contaba con todos los entrenadores como participes de la programación (7 en total), lo cual era muy reconfortante. Al final, fue el boca a boca lo que hizo que la aportación ofrecida ganara aceptación con el paso del tiempo. Tras muchas observaciones durante los entrenamientos -el autor también era psicólogo de chándal (Roffé, 2007)- y un esfuerzo en comprender la planificación de los entrenadores, se partió de la línea temporal de la periodización del entrenamiento físico-técnico de la temporada (inicio en septiembre y final en julio), como referencia. Con esa lógica, empezando desde septiembre, se realizaba una sesión grupal a la semana con cada sector (saltos, lanzamientos, fondo y velocidad), donde se abordaban los aprendizajes de las distintas variables psicológicas relacionadas con el rendimiento deportivo (por ejemplo, el establecimiento de metas, el control de los auto diálogos, la atención, gestión de la ansiedad, la imaginería o relajación). Realmente, la orientación del desempeño ha sido más de corte educativo que de alto rendimiento en sí -a excepción de algún atleta destacado-, o durante el trabajo posterior con atletas ya en categoría senior de QAF. Seguramente, un trabajo más orientado al desarrollo del bienestar psicológico del deportista a través del coaching, como lo propuesto por otros autores en estas edades (García-Naveira et al. 2017). En definitiva, la idea fue desarrollar una especie de currículo educativo a través del cual explicar qué habilidades psicológicas podrían ayudar a los atletas a mejorar su rendimiento y, como se hizo referencia anteriormente, a contribuir a su desarrollo personal y bienestar psicológico (tabla 2).

Como se puede apreciar en la tabla 2, se han llevado a cabo sesiones semanales con aproximadamente dos objetivos diferentes para cada mes hasta el mes de febrero y más individualizados en la temporada de aire libre a partir de marzo. Más importante aún, al final de cada sesión se conversaba abiertamente sobre la transferencia de cada una de ellas a otros contextos de la vida, como podría ser organizarse para preparar unos exámenes o desenvolverse mejor en las relaciones interpersonales, a modo de life skills. A partir de esas sesiones se mejoraba la compresión de las necesidades que surgían en cada grupo y no había ningún problema en desviarse del tema si surgiera alguna duda en la que mereciera la pena profundizar, lo cual ocurría muy a menudo. Al final, las sesiones han sido una excusa para crear un espacio donde comunicarse, aprender juntos y forjar una identidad de grupo. Sobre los objetivos de cada bloque de sesiones que suelen coincidir con los meses del año se puede apuntar lo siguiente:

Septiembre: En este mes la clave era reconectar con los atletas tras el descanso de agosto. Dado que los entrenadores tienen que planificar la temporada (aunque realmente esto ya lo suelen hacer antes de que termine la anterior), se ponía la atención en el establecimiento de metas como hilo conductor durante estas sesiones, debido a que una buena comprensión de este concepto contribuye al compromiso y adherencia de los atletas en los entrenamientos (Morelló et al. 2018). Se ponía también el acento en los valores, en lo que es importante para ellos y cómo sus valores podían ayudarles a conseguir sus objetivos en la vida. Igualmente, se comenzaba este mes con una temática sobre "cómo piensan los campeones", introduciéndoles al entrenamiento en auto instrucciones y a patrones de pensamiento adaptativo 
Tabla 2. Programación anual de entrenamiento de distintas variables psicológicas en el atletismo en Aspire Academy 2008-2021

\begin{tabular}{|c|c|c|}
\hline \multicolumn{3}{|c|}{ Preparación psicológica en el atletismo en Aspire Academy 2008-2021 } \\
\hline Mes & Contenido de la sesión & Objetivo \\
\hline \multirow[t]{2}{*}{ Septiembre } & Establecimiento de metas & $\begin{array}{l}\text { Introducción al concepto de metas; a corto, medio, largo plazo. Metas de proceso y de resul- } \\
\text { tado. Alinear conceptos con objetivos del entrenador para cada atleta durante la temporada } \\
\text { (Morelló et al. 2018). }\end{array}$ \\
\hline & Autohabla/atención-concentración & $\begin{array}{l}\text { Concepto de atención. Técnicas de control del pensamiento: parada de auto diálogo, auto } \\
\text { instrucciones, autoafirmaciones (Mora-Mérida y Díaz-Ocejo, 2008; Díaz-Ocejo et al. 2013). }\end{array}$ \\
\hline \multirow[b]{2}{*}{ Octubre } & Técnicas de relajación & $\begin{array}{l}\text { Técnica de relajación muscular (Jaenes y Caracuel, 2016). } \\
\text { Técnicas de respiración (Módulo de Respiración de biofeeback, Vienna Test System, Schu- } \\
\text { fried 2000Xpert, Schuhfried, 2013). }\end{array}$ \\
\hline & Imagninería & $\begin{array}{l}\text { Concepto (Gregg y Hall, 2018) y práctica (Koehn y Díaz-0cejo, 2016; Díaz-Ocejo y Foun- } \\
\text { toulakis, 2011) y en biofeedback con electromiograma (EMG) y neurofeedback (Mindroom), } \\
\text { (Ming-Yang y Tsung-Min, 2020). (Cheng et. al, 2020) } \\
\text { Sesiones video-asistidas con unidad de biomecánica (vídeos personalizados en aplicación } \\
\text { móvil para cada atleta). }\end{array}$ \\
\hline \multirow{2}{*}{ Noviembre } & Control nivel de activación & $\begin{array}{l}\text { Concepto y práctica de respuesta galvánica de la piel (biofeeback, Vienna Test System, Schu- } \\
\text { fried 2000Xpert), (Ming-Yang y Tsung-Min, 2020). (Cheng et. al, 2020) }\end{array}$ \\
\hline & Ansiedad precompetitiva & $\begin{array}{l}\text { Concepto y aplicaciones prácticas con estrategias para: ansiedad cognitiva (estrategias cog- } \\
\text { nitivas), ansiedad somática (estiramientos, respiración diafragmática) (Hanton et al. 2008). }\end{array}$ \\
\hline Diciembre & Rutinas pre-ejecución & $\begin{array}{l}\text { Integrar aprendizajes y estrategias en secuencias de rutinas pre-ejecución y creación de } \\
\text { hábitos (Hazell et al. 2014). }\end{array}$ \\
\hline \multirow[b]{2}{*}{ Enero } & Autoconfianza & Concepto y fuentes que contribuyen al desarrollo de la autoconfianza (Hays et al. 2009). \\
\hline & Concentración de Invierno & $\begin{array}{l}\text { Observaciones por parte del psicólogo en competición (Holder y Winter, 2017) y fomento del } \\
\text { desarrollo de life skills en convivencia en el hotel. }\end{array}$ \\
\hline \multirow{2}{*}{ Febrero } & Reflective practice & $\begin{array}{l}\text { Repaso y análisis del insight individualizado del rendimiento en competición durante la } \\
\text { concentración (Neil et al. 2013). }\end{array}$ \\
\hline & Planes individualizados & $\begin{array}{l}\text { Sesiones grupales de repaso de estrategias y focalización más específica en temas indivi- } \\
\text { duales a reforzar }\end{array}$ \\
\hline $\begin{array}{l}\text { Marzo } \\
\text { Abril } \\
\text { Mayo }\end{array}$ & $\begin{array}{l}\text { Implementación/monitorización de } \\
\text { estrategias en competición }\end{array}$ & $\begin{array}{l}\text { Seguimiento más personalizado y focalizado en aquellos atletas que competirán en cam- } \\
\text { peonatos asiáticos y mundial de atletismo junior }\end{array}$ \\
\hline $\begin{array}{l}\text { Junio } \\
\text { Julio }\end{array}$ & $\begin{array}{l}\text { Concentración de verano y puesta a } \\
\text { punto competiciones internacionales }\end{array}$ & Observaciones, monitorización y convivencia en alta competición \\
\hline
\end{tabular}

al contexto de rendimiento (Mora-Mérida y Díaz-Ocejo, 2008). Sin el cual, por ejemplo, no tendrán éxito al focalizar la atención o regular la ansiedad precompetitiva y que serán objeto de otras sesiones más adelante.

- Octubre: Se considera el aprendizaje en relajación como base para posteriormente abordar estrategias más complejas como la imaginería o, evidentemente, la gestión de la ansiedad. De ahí la idoneidad de haberlo presentado en octubre al comienzo de la temporada de entrenamiento. Para ese propósito, se entrenaba a los atletas a relajarse siguiendo las premisas básicas de la relajación muscular, aunque prescindiendo de los ejercicios de grupos musculares pequeños con los que no están tan familiarizados los deportistas jóvenes, tal y como se ha empleado anteriormente en el atletismo (Jaenes y Caracuel, 2016).

Igualmente, se les entrenaba en respiración diafragmática a través de la tecnología de biofeedback (Vienna Test System, Schufried 2000Xpert), ya que el "módulo de respiración” permitía calibrar el ciclo de respiración óptimo de cada atleta, ofreciendo una gráfica de retroalimentación en vivo con una pantalla situada en frente del mismo (Schuhfried, 2013). Posteriormente, se introducía el concepto de imaginería y se les animaba a practicar 


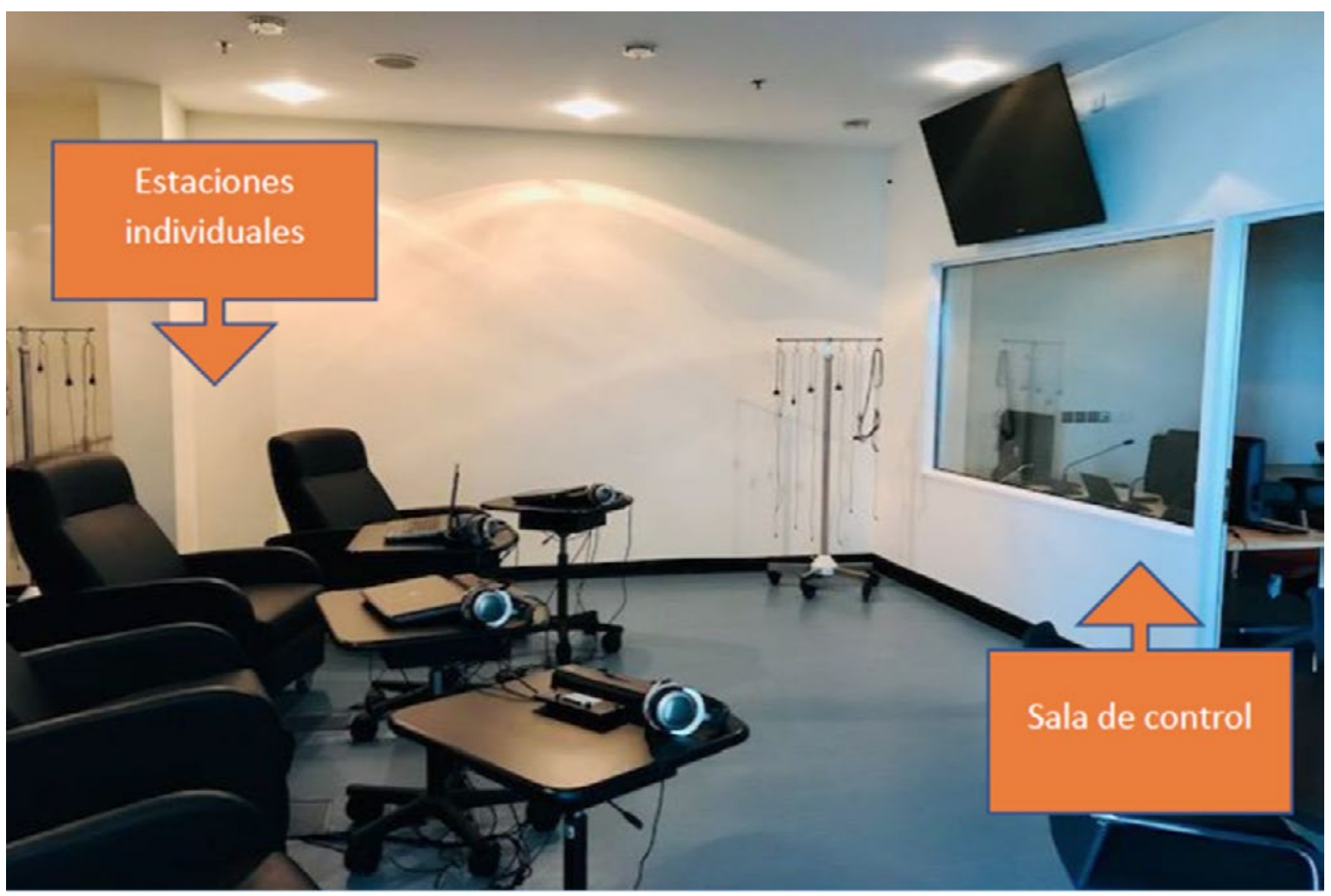

Figura 1. Diseño del Mindroom en Aspire Academy, con cuatro estaciones individuales (a la izquierda de la imagen) y una sala de control (a la derecha de la imagen). Fuente: Elaboración propia

este aprendizaje tal y como se recomienda en experiencias similares en el atletismo a estas edades (Gregg y Hall, 2018) o más próximas a nuestro contexto (Koehn y Díaz-Ocejo, 2016; Díaz-Ocejo y Fountoulakis, 2011) y para lo que también se introducía el empleo de electromiograma (EMG) y neurofeeback en el laboratorio de psicofisiología de Aspire denominado Mindroom (figura 1). Afortunadamente, dado el suculento presupuesto de Aspire, en 2013 se completó la creación de un laboratorio Mindroom de biofeedback y neurofeeback, siguiendo el concepto desarrollado en Canadá por la tecnología Thought Technology, Ltd. Este laboratorio está hecho a imagen y semejanza de la adaptación de este concepto al Mindroom auspiciado por Bruno De Michelis en el AC Milán y el Chelsea FC (Beauchamp et al. 2012). El Mindroom cuenta con cuatro estaciones individuales de bio/neurofeedback y una sala de control (figura 1). Esta tecnología ha permitido realizar intervenciones con algunos atletas destacados a través de mediciones de parámetros tanto de biofeedback como de neurofeedback, como, por ejemplo, observar respuestas de ondas cerebrales a través del uso de electroencefalograma (EEG), o el empleo de electromiograma (EMG) durante la práctica en imaginería (Ming-Yang y Tsung-Min, 2020; Cheng et. al, 2020). Para este propósito, se entrenaba al atleta en imaginería en sesiones personalizadas y otras asistidas con el uso de videos individualizados desarrollados por la uni- dad de Biomecánica sobre su rendimiento (por ejemplo, una batida en un salto de altura o una fase determinada en un lanzamiento) a los que se añadían palabras clave asociadas con momentos concretos de ejecución. Estas claves guiaban el proceso de imaginería (mediado por auto-instrucciones) para cada atleta. Podían visionarse y escucharse en sus teléfonos móviles a través de una aplicación creada para ese propósito. Igualmente, se añadía el sonido real de cada zancada, el de la batida o el sonido del gesto final del lanzamiento en tiempo real o ralentizado. Tomando como referencia esta experiencia en el entrenamiento en imaginería y aunque el autor considera que todavía sabemos muy poco sobre cómo integrar la información sobre todo de neurofeedback al terreno aplicado en nuestro ámbito de trabajo, las neurociencias tienen el futuro garantizado en el deporte de rendimiento.

Noviembre: Ya en preparación para el manejo de la ansiedad precompetitiva en las competiciones venideras, se introducía a los atletas en el concepto de nivel de activación o arousal y se les enseñaba a identificar lo que sería su nivel óptimo de activación. Esto, tanto a nivel cognitivo como somático. Así, se llevaba a cabo una monitorización semanal de sus niveles óptimos subjetivos de activación durante distintos momentos de los entrenamientos, para más tarde compararlos con los que se obtenían en situación de competición. En este sentido, se 
les enseñaba a comprender el concepto de arousal a través del empleo de la respuesta galvánica de la piel (MingYang y Tsung-Min, 2020), con tecnología de biofeeback, (Vienna Test System, Schufried 2000Xpert). Posteriormente, se les explicaba el concepto de ansiedad competitiva (Hanton et al. 2008) y se les instruía en el aprendizaje de estrategias de regulación de ansiedad cognitiva (estrategias cognitivas como la parada del pensamiento, autoinstrucciones o autoafirmaciones) y para la ansiedad somática (estiramientos, respiración diafragmática).

- Diciembre: Una vez establecidos los aprendizajes en algunas de las variables psicológicas más importantes de cara al rendimiento deportivo, se enseñaba a los atletas a integrarlas según sus necesidades dependiendo de la especialidad en el atletismo. Por ejemplo, en los concursos, donde existe tiempo para organizar el pensamiento y la conducta de "pre-ejecución", es crucial crear una secuencia lógica de organización de esta. Así, por ejemplo, tener una meta clara, gestionar el nivel de activación y regular la ansiedad, focalizar la atención a través del empleo de auto instrucciones y tranquilizarse dándose ánimos, puede suponer una secuencia lógica para afrontar el siguiente reto. Aquí se les animaba a individualizar las rutinas y a mantenerlas consistentes para que fueran más manejables y automatizadas en situación de competición (Hazell et al. 2014).

- Enero: Una vez adquirían conocimientos y práctica de estas habilidades básicas en los entrenamientos, la idea era prepararlos para ponerlas en práctica en las competiciones de la concentración que se realizaban cada año en Sudáfrica en enero. La última variable que se abordaba era el concepto de autoconfianza dada su relevancia antes de la competición deportiva (Hays et al. 2009). Del mismo modo, ya de viaje, se aprovechaba la oportunidad de convivencia durante la concentración para fomentar las "habilidades para la vida" (life skills), repasar sus metas y reflexionar sobre los valores, la transferencia de los conocimientos a otras facetas de la vida, cómo no, la observación del desenvolvimiento real de los atletas en situación de competición (Holder y Winter, 2017). Se realizaban sesiones de grupo de "repaso" en el hotel y se abordaban situaciones que surgían in situ (por ejemplo y principalmente; conflictos interpersonales, adherencia a horarios o la higiene del sueño).

Febrero: De vuelta a Catar, se organizaban sesiones individualizadas entre el atleta, entrenador y el psicólogo deportivo con el objetivo de analizar lo sucedido en competición, desde el punto de vista de resultados y de mejoras que podían realizarse a nivel de rendimiento psicológico. El objetivo de estas sesiones era realizar un ejercicio de reflective practice (una reflexión o insight sobre los momentos determinantes de la preparación para la competición o durante la de propia competición) dirigido por cada atleta (en ocasiones lo anotan en un diario durante las competiciones), para a partir del cual confeccionar un plan más individualizado en base a las necesidades específicas que el entrenador y el atleta creen convenientes (Neil et al. 2013). A partir de aquí, se realizaba un planteamiento más de alto rendimiento, abordándose, por ejemplo, casos específicos de ansiedad precompetitiva, focalización atencional, o una mejor gestión de las rutinas pre-ejecución

Marzo, abril, mayo: En estos meses de competición local en Catar, se priorizaba la monitorización del empleo de las estrategias seleccionadas para la mejora de la parcela psicológica. Esto se hacía con medidas de autorregistro en ocasiones creadas para las necesidades específicas de cada deportista y observaciones in situ por parte del psicólogo del deporte. Eran meses donde la realidad de las necesidades del atleta era muy cambiante, fruto de las competiciones ya casi semanales, la carga del entrenamiento, la ocurrencia de lesiones o el resultado de las competiciones mismas. Aquí ya no habría sesiones grupales, sino individuales. Estos eran meses de mucha flexibilidad por parte del autor, de escuchar mucho, de prestar mucha atención a todo lo que rodeaba al atleta y la competición y de saber obviar o eliminar lo redundante y que no serviría para los Campeonatos del Mundo Junior o asiático en verano.

- Junio, julio: Aquí la preparación se adentraba en el objetivo que se tenía desde septiembre. En junio se iba de concentración a Europa unas siete semanas, donde se culminaba la preparación para la competición internacional. Sólo iban los atletas que tenían la marca mínima para los campeonatos asiáticos o del mundo. El foco estaba en establecer una rutina de alto rendimiento focalizada en la preparación para rendir al máximo en julio. Los horarios eran ya más estrictos que en la concentración de invierno, se cuidaba más la dieta, se gestionaban mejor los descansos y se elegía cualquier momento que fuera adecuado para escuchar al atleta. Normalmente se trataba de unos seis o siete atletas, a quienes ya se conocía muy bien y con quienes tan solo había que estar presentes para ayudarles a "hilar más fino" en algunas de las estrategias que se estuviese refinando. Aquí era cuando un paseo por el parque, una cena, o un café en la recepción de un hotel servían como momentos no previstos o planificados para repasar estrategias psicológicas, compartir vivencias, miedos o ansiedades con ellos. Estos eran momentos muy válidos en la práctica profesional, 
para los que era necesario haber adquirido suficiente confianza mutua como para que fueran así de significativos. Por otro lado, en general, lo que sí solía ocurrir era un aumento de la ansiedad general del grupo a medida que se acercaba la competición internacional. Era ahí cuando el entorno se volvía más impredecible y tenso, algo parecido a ese hábitat natural aludido anteriormente, donde la labor del psicólogo o psicóloga del deporte cumple una función esencial como "amortiguadores" de esa presión ambiental tan presente en la alta competición.

\section{Consideraciones culturales}

Tal y como se comentó al comienzo del artículo, parece imprescindible dedicar una sección a la cultura. Aunque dada la complejidad e importancia del tema, se le podría brindar un artículo completo. Se ha mencionado anteriormente que, por ejemplo, una de las limitaciones del trabajo en Aspire ha sido la falta de instrumentos de medición baremados en población árabe en PD. Sin embargo, la limitación más importante de esta experiencia personal en Catar ha sido sin duda la barrera cultural. De acuerdo con Parham (2005), el psicólogo del deporte que no comprenda la cultura en la que se encuentra, tendrá muchas dificultades para tener éxito con los deportistas con quienes trabaje. En efecto, la diferencia cultural entre occidente y Catar es tan grande, que deben tenerse en cuenta, al menos, ciertas consideraciones. Se abordará esta cuestión en torno a dos conceptos generales; el contexto cultural y algunas claves para entender al deportista catarí.

\section{Contexto cultural en Catar}

En relación con el contexto cultural y desde un punto de vista puramente antropológico, Catar sería un ejemplo del denominado "relativismo cultural" (Kottak, 2007). Es decir, el fundamento sería que toda cultura es relativa y todo es relativo a la cultura. Con esta asunción, las cosas en esta parte del mundo son como son porque siempre se han hecho así, no son ni mejores ni peores que otra cultura. No existe una moralidad superior o internacional de carácter universal, puesto que es pertinente respetar las reglas éticas de cada cultura. Como consecuencia de ello, en Catar no se cuestionan o debaten asuntos relacionados con su tradición, cultura o religión, simplemente, se aceptan como son. Lo cual explicaría, por ejemplo, que la Academia no tenga en consideración la admisión de chicas y que nadie proteste por esta circunstancia. Esto, desde un punto de vista occidental, significaría un inmovilismo y una intromisión de los derechos culturales en los derechos individuales. Por otro lado, la cultura catarí, como otros países asiáticos, es de tipo colectivista. Sin embargo, a diferencia de otros países del continente, también se trata de una cultura no competitiva. Es decir, no es una sociedad que prime la individualidad, la creatividad, el pensamiento crítico, el liderazgo, ni la competitividad sino, más bien, la conformidad con la cultura, la religión y las normas sociales establecidas. Además, de acuerdo con Galloway (2009) sobre su experiencia como psicólogo del deporte en Kuwait, el profesional occidental observará cómo establecer relaciones sociales y vínculos afectivos con sus deportistas puede conllevar un proceso más lento. Esto puede representar una barrera a la hora de establecer rapport o un vínculo terapéutico por parte del psicólogo occidental. Existe evidencia de que, en estas culturas de origen nómada, las relaciones de apego se forman de un modo distinto a occidente, al ser la crianza de los recién nacidos una responsabilidad compartida por toda la familia, más allá que de los cuidadores principales (Oates et al. 2009). Por ello, no se formaría un vínculo afectivo principal exclusivamente con la madre (o el cuidador principal), como suele ocurrir en occidente, sino más bien compartido con varios miembros de la familia, que suelen ser muy numerosas. Este modelo de apego supondría que, en la edad adulta, los deportistas cataríes se relacionen con muchas personas, aunque de un modo que puede parecer algo distante, a los ojos del profesional occidental. Este modelo también explicaría una cierta inclinación hacia la dependencia interpersonal, más que al desarrollo de la autonomía como la conocemos en occidente. Igualmente, el valor de la familia cobra una dimensión especial. En temas de relevancia, por ejemplo, el padre será quien tome la decisión final y no habrá forma de rebatirlo. Asimismo, los padres se involucran muy poco en la formación deportiva de sus hijos y mucho menos en el de las hijas. Esto también tiene gran repercusión al abordar los casos clínicos, en una cultura donde la psicología es todavía un tabú. Ante un "problema" de tipo psicológico, normalmente, la familia acudirá a un imán de una mezquita en primera instancia, antes de consultar con un psicólogo. En esta experiencia, siempre se ha contado con un compañero catarí para contactar con la familia primero y se ha respetado su decisión en cuanto a cómo actuar. En otras ocasiones, la familia acudirá a un psicólogo de fe musulmana, quien probablemente abordará el problema desde la "psicoterapia islámica", basada en el rezo.

\section{Algunas claves para la intervención con el deportista catarí}

En este contexto, el trabajo con estos deportistas puede suponer un reto para el profesional occidental. Por ejemplo, 
en relación con la comunicación, el saludo es muy importante y al que hay que dedicarle atención y tiempo. En un mismo encuentro, el catarí puede preguntar tres o cuatro veces "¿cómo estás?" o "¿cómo está la familia?", mientras que el occidental puede verse confundido por tal repetición. Al catarí no le gusta decir "no", simplemente, no está considerado ser de buena educación decir no. Esto puede llevar a confusión ya que en raras ocasiones dirán "no", en su lugar, dirán Inshallah, que en árabe significa "si Alá quiere", pero que no es ni un sí, ni un no. Con lo cual, habrá que esperar para saber con seguridad cuál fue la intención de su respuesta. En cuanto al lenguaje corporal, por ejemplo, el no establecer contacto con la mirada significa respeto hacia el entrenador, quien puede interpretarlo como falta de respeto al no mirarle a la cara cuando hablen. Más importante aún, sobre todo en deportes de equipo, hay que tener en cuenta a los que el autor denomina "líderes invisibles". En Catar, no todas las familias son iguales y algunos apellidos están mejor considerados socialmente dada su influencia o proximidad con la familia real. Si un jugador pertenece a una de estas familias, gozará automáticamente de un estatus especial en el equipo. Un entrenador que riña públicamente o ponga en evidencia a uno de estos líderes se arriesga a ofender el honor de su familia, lo que, desafortunadamente, tiene poca solución una vez ya haya ocurrido. En estos casos, lo ideal es tratar temas delicados en privado con estos "líderes", para evitar mayores complicaciones. Igualmente, dado que la religión penetra en todas las facetas de la vida en Catar, un jugador que sea capaz de recitar más versos del Corán también gozará de un estatus especial en el grupo, aunque sea un recién llegado o no tan talentoso para el deporte. De nuevo, un entrenador que nos sea consciente de ello puede tener muchos problemas para comprender la dinámica de grupo en su equipo. Por otro lado, la percepción del tiempo en esta parte del mundo es diferente. Decir "ahora", puede interpretarse como "en unos quince minutos" fácilmente, algo complicado de comprender para el recién llegado de occidente. Esta percepción de la temporalidad afecta, igualmente, al trabajo como psicólogos del deporte. Muy a menudo ha ocurrido que, al abordar el establecimiento de metas con ellos, mayormente se interesan por las metas a corto plazo, con lo que la planificación a largo plazo puede verse comprometida.

Finalmente, de acuerdo con Azkargorta (2004) en cuanto a la relación existente entre la forma de vivir y la competición deportiva, en Catar, que posiblemente es el país más rico del mundo gracias a su gas natural líquido, las personas crecen sin necesidad real de sacrificarse para lograr resultados superiores, del modo que lo concebimos en occidente. Esto, indudablemente, supone una limitación para desarro- llar una mentalidad resiliente. A consecuencia de ello, lo sugerido por la PD occidental, como sería fomentar la dureza mental, la toma de decisiones o la iniciativa del deportista, está, marcadamente, en contraposición a su forma de crecer. Más bien, la labor será acompañarlos y guiarlos durante un largo aprendizaje hacia un delicado equilibrio entre el desarrollo de la propia agencia o autonomía -como se entiende en occidente-, junto con el respeto a esa tendencia hacia la dependencia interpersonal y el reloj social (por ejemplo, los cataríes se casan con alrededor de veinte a veinticinco años), tan arraigado en Catar. No tener en cuenta todas estas consideraciones por parte del entrenador, o el psicólogo del deporte, complicará mucho su trabajo, algo que se puede avalar con esta experiencia.

\section{Discusión}

Como se ha expuesto, el devenir de esta práctica profesional en Aspire Academy ha sido una aventura inmensamente enriquecedora. Ha estado marcada por cambios y adaptaciones a través de los distintos equipos de dirección, sin duda cimentada en numerosos errores a lo largo de este desempeño profesional en la PD aplicada. La piedra angular de esta experiencia ha girado indudablemente alrededor de la cultura, que lo envuelve todo y sin la que no se alcanzaría a comprender nada. Así, la cultura puede suponer el principal reto para la práctica profesional como psicólogos y psicólogas del deporte en Catar. Está claro que llegar a comprender al deportista catarí puede llevar tiempo, al igual que comprender su cultura. La comunicación, el lenguaje corporal, el ritmo para irse conociendo, las normas sociales y un largo etcétera, suponen limitaciones a la que todo occidental se ve abocado al Ilegar a Catar. De igual modo, la falta de experiencias aplicadas en el deporte infanto-juvenil, más concretamente en el atletismo, más aún en esta cultura, ha supuesto otra de las limitaciones a lo largo de estos años. Son bien conocidas las intervenciones en PD con deportistas de élite, pero hacen falta más experiencias aplicadas con población adolescente. En Catar, dada la carencia de experiencias anteriores en materia de PD a la aparición de Aspire, se optó por ir configurando una programación en PD basada principalmente en las necesidades que tenían los entrenadores, más que tomar como referencia otras experiencias previas en otros países de una cultura diferente. A estas limitaciones se añade la carencia de instrumentos de recogida de información desde la PD en lengua árabe, aunque esta experiencia posiblemente demuestre que existen otros abordajes para suplir esta carencia, tal y como se ha descrito con el empleo de la plataforma VTS, la creación de una entrevista 
ajustada al contexto de Aspire en materia de captación de talentos deportivos y evaluación del perfil de rendimiento del deportista. Por otro lado, también en materia de captación y desarrollo de talentos deportivos, se ha podido presenciar y participar en el desarrollo de deportistas desde los 12 años hasta la alta competición en categoría senior, lo cual ha sido un auténtico regalo. Ello ha permitido comprobar que, efectivamente, por más que se insista, es casi imposible predecir quién será un campeón a esas edades tan tempranas. En ese sentido, quizá debería darse más relevancia a las ganas del deportista y su entorno que al talento puro. O quizá, el talento es precisamente la combinación de ambas, las ganas y el entorno en el que se desarrolla el deportista. Ciertamente, esta experiencia ha permitido el desarrollo de una programación a lo largo de los años que ha permitido adaptar las bases del conocimiento actual en materia de habilidades psicológicas, al contexto de Aspire. Se ha aprendido que el hilo conductor de esa contribución de la PD depende enormemente de los entrenadores y del desarrollo de esa relación mutua entre ellos, los deportistas y el psicólogo del deporte. En definitiva, se ha aprendido que lo que se hace es sumergirse en un proceso dinámico de enriquecimiento recíproco y de crecimiento personal y se hace con la excusa del deporte.

\section{Referencias}

Abbott, A. y Collins, D. (2002). A theoretical and empirical analysis of a 'state of the art' talent identification model. High Ability Studies, 13(2), 157-178. http://dx.doi. org/10.1080/1359813022000048798

Azkargorta, X. (2004). Se juega como se vive: Un análisis psicosocial del fútbol profesional. Cuadernos de Psicología del Deporte, 4, (1-2), 53-57. https://revistas.um.es/cpd/article/view/112511

Beauchamp, M. K., Harvey, R. H. y Beauchamp, P. H. (2012). An Integrated Biofeedback and Psychological Skills Training Program for Canada's Olympic Short-Track Speedskating Team. Journal of Clinical Sport Psychology, 6(1), 67-84. https://doi. org/10.1123/icsp. 6.7.67

Cheng, M. Y. y Hung, T. M. (2020). Biofeedback and neurofeedback for mental skills training in sports. Advancements in Mental Skills Training, Routledge. https://doi.org/10.4324/9780429025112-14

De Andrade, M. O. C., González-Víllora, S., Casanova, F. y Teoldo, I. (2020). The attention as a key element to improve tactical behavior efficiency of young soccer players. Revista de Psicología del Deporte, 29(2), 47-55.

De Rond, M. (2012). There is an I in Team: What Elite Athletes and Coaches Really Know About High Performance. Harvard Business Review Press.

Díaz-Ocejo, J. y Fountoulakis, C. (2011). The effects of a 6-month intervention on student-athletes' reactive stress tolerance. En Qatar Foundation Annual Research Forum Proceedings (No. 2011, p. AHP2). Bloomsbury Qatar Foundation Journals.
Díaz-Ocejo, J., Kuitunnen, S. y Mora-Mérida, J. A. (2013). An intervention to enhance the performance of a 3000 metre steeplechase athlete with the use of segmentation and self-talk. Revista de Psicología del Deporte, 22(1), 87-92.

Galloway, S. (2009). A Canadian sport psychologist in Kuwait. En R. J. Shinke y S. J. Hanrahan (Eds.), Cultural Sport Psychology (pp. 153-164). Human Kinetics.

García-Naveira, A. (2016). Área de Psicología de la Real Federación Española de Atletismo: programación y desempeño profesional. Cuadernos de Psicología del Deporte, 16(1), 251-258. https://revistas.um.es/cpd/article/view/254611

García-Naveira, A., García-Mas, A., Ruiz-Barquín, R. y Cantón, E. (2017). Programa de intervención basada en el coaching en jóvenes deportistas de alto rendimiento y su relación con la percepción de bienestar y salud psicológica. Revista de Psicología del Deporte, 26(2), 37-44.

García-Naveira, A. (2018). Autoeficacia y rendimiento en jugadores de fútbol. Cuadernos de Psicología del Deporte, 2(18), 66-78. https://revistas.um.es/cpd/article/view/319341

Gregg, M. y Hall, C. (2018). Imagery as a skill: Longitudinal analysis of changes in motivational imagery. Imagination, Cognition and Personality, 37(4), 448-457. https://doi. org/10.1177/0276236617735101

Hanton, S., Neil, R. y Mellalieu, S. D. (2008). Recent developments in competitive anxiety direction and competition stress research. International Review of Sport and Exercise Psychology, 1(1), 45-57. https://doi.org/10.1080/17509840701827445

Hazell, J., Cotterill, S. T. y Hill, D. M. (2014). An exploration of pre-performance routines, self-efficacy, anxiety and performance in semi-professional soccer. European Journal of Sport Science, 14(6), 603-610. https://doi.org/10.1080/17461391.2014.888484

Hays, K., Thomas, O., Maynard, I. y Bawden, M. (2009). The role of confidence in world-class sport performance. Journal of Sports Sciences, 27(11), 1185-1199. https://doi. org/10.1080/02640410903089798

Hackfort, D., Kilgallen, C. and Hao, L. (2009). The action theory-based mental test and training system (MTTS). En T. M. Hung, R. Lidor y D. Hackfort (Eds.), Psychology of sport excellence: International perspectives on sport and exercise psychology (Vol. 1, pp. 15-24). Fitness Information Technology.

Holder, T. y Winter, S. (2017). Experienced practitioners' use of observation in applied sport psychology. Sport, Exercise, and Performance Psychology, 6(1), 6-19. https://doi.org/10.1037/ spy0000072

Horn, T. S. (2004). Developmental Perspectives on Self-Perceptions in Children and Adolescents. En M. Weiss (Eds.), Developmental Sport and Exercise Psychology: A Lifespan Perspective. (pp. 1-26). Fitness Information Technology.

Jaenes, J. C., Rivera, M. y Hechaverria, R. (2012). Intervención psicológica en los XXI Juegos Centroamericanos y del Caribe. Revista de Psicología del Deporte, 21(1), 177-181.

Jaenes, J.C. y Caracuel, J. C. (2016): Maratón: Preparación psicológica para el entrenamiento y la competición. ( $2^{a}$ Ed.). Almuzara

Jaenes, J. C., García Mas, A., Rivera, M. y Hechavarria, R. (2019). Intervención forense y psicológica en un atleta de élite con psicopatología sobrevenida. Revista Andaluza de Medicina del Deporte, 12(1), 50-52. 
Kilgallen, C. (2013). Developing Sporting Talent in Qatar. En M. B. Sualyem, S. O'Connor y D. Hassan, (Eds), Sport Management in the Middle East: a Case Study Analysis (pp. 173-193). Routledge.

Kiss, B. y Balogh, L. (2019). A study of key cognitive skills in handball using the Vienna test system. Journal of Physical Education and Sport, 19(1), 733-741.

Koehn, S. y Díaz-Ocejo, J. (2016). Imagery intervention to increase flow state: A single-case study with middle-distance runners in the state of Qatar. International Journal of Sport and Exercise Psychology. https://doi.org/10.1080/1612197X.2016.1187653

Kottak, C. (2007). Introducción a la Antropología Cultural. McGraw-Hill.

Ming-Yang, C. y Tsung-Min, H. (2020). Biofeedback and Neurofeedback for Mental Skills Training in Sports. En R. J. Schinke, T. Schack y A. Papaioannou (Eds), International Perspectives on Key Issues in Sport and Exercise Psychology Series (pp.149-163). Routledge.

Mora-Mérida, J. A. y Díaz-Ocejo, J. (2008). Control del Pensamiento y sus Estrategias en el Deporte. EOS.

Morelló, E., Bert, B. y Navarro, S. (2018). Establecimiento de objetivos en el curriculum formativo de los futbolistas. Revista de Psicología aplicada al Deporte y al Ejercicio Físico, 3(1), Artículo e3. https://doi.org/10.5093/rpadef2018a7

Neil, R., Cropley, B., Wilson, K. y Faull, A. (2013). Exploring the value of reflective practice interventions within applied sport psychology: Case studies with an individual athlete and a team. Sport and Exercise Psychology Review, 9(2), 42-56.

Nigro, P. y Androetto, M (2020). Club Atlético River Plate: claves psicológicas de un campeón de América. Revista de Psicología Aplicada al Deporte y al Ejercicio Físico, 5(2), Artículo e10, 1-7. https://doi.org/10.5093/rpadef2020a13

Nitsch, J. R. y Hackfort, D. (1981). Streß in Schule und Hochschule-eine handlungspsychologische Funktionsanalyse [Estrés en el colegio y la universidad: Un análisis funcional basado en la teoría de la acción]. En J. R. Nitsch (Ed.), Stress. Theorien, Untersuchungen, Maßnahmen (pp. 263-311). Huber.

Oates, J., Lewis, C. y Lamb, M. E. (2009). Parenting and attachment. En S. Ding y K. Littleton (Eds), Children's Personal and Social Development (pp. 11-52). Blackwell Publishing: The Open University.
Ong, N. C. (2017). Reactive stress tolerance in elite athletes: Differences in gender, sport type, and competitive level. Cognitie, Creier, Comportament/Cognition, Brain, Behavior, 21 (3), 189-202. https://doi.org/10.24193/cbb.2017.21.11

Parham, W. D. (2005). Raising the bar: Developing an understanding of athletes from racially, culturally and ethnically diverse backgrounds. En M. B. Andersen (Ed.). Sport Psychology in Practice (pp. 201-216). Human Kinetics.

Pulido-Pedrero, S. P., de la Vega Marcos, R. y García, J. P. F. (2020). Motivación de logro en deportistas de combate de élite: evaluación objetiva computerizada. Revista de Psicología del Deporte, 29(1), 75-82.

Roffé, M. (2007). Psicología del jugador de fútbol: con la cabeza hecha pelota, (3a edición). Editorial Lugar.

Roffé, M. (2016). La preparación psicológica de la Selección Nacional Absoluta de Colombia para el Mundial de Fútbol Brasil 2014. Revista de Psicología Aplicada al Deporte y al Ejercicio Físico, 2(1), Artículo e2. https://doi.org/10.5093/rpadef2017a2

Romero, J. R., Baídez, M. M. y Chirivella, E. C. (2018). Entrenamiento psicológico mediante el coaching motivacional en alto rendimiento: una experiencia en marcha atlética. Revista de Psicología Aplicada al Deporte y al Ejercicio Físico, 3(2), Artículo e14. https://doi.org/10.5093/rpadef2018a11

Ruiz de Oña, M. (2018). La mirada del psicólogo: 21 años de experiencia en el Athletic Club de Bilbao. Revista de Psicología Aplicada al Deporte y al Ejercicio Físico, 3(1), Artículo e6. https://doi. org/10.5093/rpadef2018a3

Schuhfried, G. (2013). Vienna Test System: Psychological Assessment. Moedling. Schuhfried.

Schumacher, N., Schmidt, M., Wellmann, K. y Braumann, K. M. (2018). General perceptual-cognitive abilities: Age and position in soccer. PloS one, 13(8), Artículo e0202627. https://doi. org/10.1371/journal.pone.0202627

Schumacher, N., Schmidt, M., Reer, R. y Braumann, K. M. (2019). Peripheral vision tests in sports: Training effects and reliability of peripheral perception test. International Journal of Environmental Research and Public Health, 16(24), 5001. https://doi. org/10.3390/ijerph16245001 